\title{
A Novel Numerical Technique for Two-Dimensional Laminar Flow between Two Moving Porous Walls
}

\author{
Zodwa G. Makukula, ${ }^{1}$ Precious Sibanda, ${ }^{1}$ and Sandile S. Motsa ${ }^{2}$ \\ ${ }^{1}$ School of Mathematical Sciences, University of KwaZulu-Natal, Private Bag X01, Scottsville, \\ Pietermaritzburg 3209, South Africa \\ ${ }^{2}$ Department of Mathematics, University of Swaziland, Private Bag 4, M201 Kwaluseni, Swaziland
}

Correspondence should be addressed to Precious Sibanda, sibandap@ukzn.ac.za

Received 16 February 2010; Revised 6 July 2010; Accepted 5 August 2010

Academic Editor: K. Vajravelu

Copyright (C) 2010 Zodwa G. Makukula et al. This is an open access article distributed under the Creative Commons Attribution License, which permits unrestricted use, distribution, and reproduction in any medium, provided the original work is properly cited.

\begin{abstract}
We investigate the steady two-dimensional flow of a viscous incompressible fluid in a rectangular domain that is bounded by two permeable surfaces. The governing fourth-order nonlinear differential equation is solved by applying the spectral-homotopy analysis method and a novel successive linearisation method. Semianalytical results are obtained and the convergence rate of the solution series was compared with numerical approximations and with earlier results where the homotopy analysis and homotopy perturbation methods were used. We show that both the spectral-homotopy analysis method and successive linearisation method are computationally efficient and accurate in finding solutions of nonlinear boundary value problems.
\end{abstract}

\section{Introduction}

Laminar viscous flow in tubes that allow seepage across contracting or expanding permeable walls is encountered in the transport of biological fluids such as blood and filtration in kidneys and lungs. Such flows have many other practical applications such as in binary gas diffusion, chromatography, ion exchange, and ground water movement [1-6]. In addition, flow in channels with permeable walls provides a good starting point for the study of flow in multichannel filtration systems such as the wall flow monolith filter used to reduce emissions from diesel engines introduced by Oxarango et al. in [7]. Consequently, in the past four decades a considerable amount of research effort has been expended in the study of laminar flows in rectangular domains that are bounded by permeable walls [8-15].

The equations governing such flows are generally nonlinear and in the past asymptotic techniques, and numerical methods have been used to analyze such flows and to solve the equations; for example, in the pioneering study by Berman [8] asymptotic methods were used 
to solve the steady flow problem for small suction. In the study by Uchida and Aoki [16], numerical methods were used to solve the governing nonlinear equations and to explain the flow characteristics. Majdalani and Roh [4] and Majdalani [3] studied the oscillatory channel flow with wall injection, and the resulting governing equations were solved using asymptotic formulations (WKB and multiple-scale techniques). The multiple-scale solution was found to be advantageous over the others in that its leading-order term is simpler and more accurate than other formulations, and it displayed clearly the relationship between the physical parameters that control the final motion. It also provided means of quantifying important flow features such as corresponding vortical wave amplitude, rotational depth of penetration, and near wall velocity overshoot to mention a few. Jankowski and Majdalani [12] used the same approach and drew similar conclusions about the multiple-scale solution for oscillatory channel flow with arbitrary suction. An analytical solution by means of the Liouville-Green transformation was developed for laminar flow in a porous channel with large wall suction and a weakly oscillatory pressure by Jankowski and Majdalani [13]. The scope of the problem had many limitations, for example, the study did not consider variations in thermostatic properties and the oscillatory pressure amplitude was taken to be small in comparison with the stagnation pressure. Zhou and Majdalani [17] investigated the meanflow for slab rocket motors with regressing walls. The transformed governing equation was solved numerically, using finite differences, and asymptotically, using variation of parameters and small parameter perturbations in the blowing Reynolds number. The results from the two methods were compared for different Reynolds numbers Re and the wall regression rate $\alpha$, and it was observed that accuracy of the analytical solution deteriorates for small Re and large $\alpha$. A good agreement between the solutions was observed for large values of Re. A similar analysis was done by Majdalani and Zhou [6] for moderate-to-large injection and suction driven channel flows with expanding or contracting walls.

In recent years, the use of nonperturbation techniques such as the Adomian decomposition method [18, 19]. He's homotopy perturbation method [20, 21], and the homotopy analysis method $[22,23]$ has been increasingly preferred to solve nonlinear differential equations that arise in science and engineering. Dinarvand et al. [2] solved Berman's model of two-dimensional viscous flow in porous channels with wall suction or injection using both the HAM and the homotopy perturbation method (HPM). They concluded that the HPM solution is not valid for large Reynolds numbers, a weakness earlier observed in the case of other perturbation techniques. Using the homotopy analysis method, $\mathrm{Xu}$ et al. [24] developed highly accurate series approximations for two-dimensional viscous flow between two moving porous walls and obtained multiple solutions associated with this problem. The multiple solutions associated with this problem were also reported by Zarturska et al. [25]. Although the homotopy analysis method is a reliable and efficient semianalytical technique, it however suffers from a number of limiting assumptions such as the requirements that the solution ought to conform to the so-called rule of solution expression and the rule of coefficient ergodicity. A modification of the homotopy analysis method, see Motsa et al. [26, 27], seeks to produce a more efficient method while also addressing the limitations of the HAM. In this paper, we use the spectral homotopy analysis method to solve the nonlinear differential equation that governs the flow of a viscous incompressible fluid in a rectangular domain bounded by two permeable walls. The problem is also solved using a new and highly efficient technique, the successive linearisation method (see $[28,29]$ ) so as to independently corroborate and validate the SHAM results. The results are also compared with numerical approximations and the recent results reported in $\mathrm{Xu}$ et al. [24] and Dinarvand and Rashidi [30]. 


\section{Governing Equations}

Consider two-dimensional laminar, isothermal, and incompressible viscous fluid flow in a rectangular domain bounded by two permeable surfaces that enable the fluid to enter or exit during successive expansions or contractions. The walls are placed at a separation $2 a$ and contract or expand uniformly at a time-dependent rate $\dot{a}(t)$. The governing Navier-Stokes equations are given in Majdalani et al. [31] as

$$
\begin{gathered}
\frac{\partial \widehat{u}}{\partial \widehat{x}}+\frac{\partial \widehat{v}}{\partial \widehat{y}}=0, \\
\frac{\partial \widehat{u}}{\partial t}+\widehat{u} \frac{\partial \widehat{u}}{\partial \widehat{x}}+\widehat{v} \frac{\partial \widehat{u}}{\partial \widehat{y}}=-\frac{1}{\rho} \frac{\partial \widehat{p}}{\partial \widehat{x}}+v \nabla^{2} \widehat{u}, \\
\frac{\partial \widehat{v}}{\partial t}+\widehat{u} \frac{\partial \widehat{v}}{\partial \widehat{x}}+\widehat{v} \frac{\partial \widehat{v}}{\partial \widehat{y}}=-\frac{1}{\rho} \frac{\partial \widehat{p}}{\partial \widehat{y}}+v \nabla^{2} \widehat{v},
\end{gathered}
$$

where $\widehat{u}$ and $\widehat{v}$ are the velocity components in the $\widehat{x}$ and $\widehat{y}$ directions, respectively, $\widehat{p}, \rho, v$ and $t$ are the dimensional pressure, density, kinematic viscosity, and time, respectively. Assuming that inflow or outflow velocity is $v_{w}$, then the boundary conditions are

$$
\begin{gathered}
\widehat{u}(\widehat{x}, a)=0, \quad \widehat{v}(a)=-v_{w}=-\dot{a} / c, \\
\frac{\partial \widehat{u}}{\partial \widehat{y}}(\widehat{x}, 0)=0, \quad \widehat{v}(0)=0, \quad \widehat{u}(0, \widehat{y})=0,
\end{gathered}
$$

where $c\left(=\dot{a} / v_{w}\right)$ is the injection or suction coefficient. Introducing the stream function $\widehat{\psi}=$ $v \hat{x} \widehat{F}(y, t) / a$ and the transformations

$$
\psi=\frac{\widehat{\psi}}{a \dot{a}}, \quad u=\frac{\widehat{u}}{\dot{a}^{\prime}}, \quad v=\frac{\widehat{v}}{\dot{a}}, \quad x=\frac{\widehat{x}}{a}, \quad y=\frac{\widehat{y}}{a}, \quad F=\frac{\widehat{F}}{\operatorname{Re}},
$$

Majdalani et al. [31] and Dinarvand and Rashidi [30] showed that (2.1)-(2.3) reduce to the normalized nonlinear differential equation

$$
F^{I V}+\alpha\left(y F^{\prime \prime \prime}+3 F^{\prime \prime}\right)+\operatorname{Re}\left(F F^{\prime \prime \prime}-F^{\prime} F^{\prime \prime}\right)=0,
$$

subject to the boundary conditions

$$
\begin{aligned}
& F=0, \quad F^{\prime \prime}=0, \quad \text { at } y=0, \\
& F=1, \quad F^{\prime}=0, \quad \text { at } y=1,
\end{aligned}
$$

where $\alpha(t)=\dot{a} a / v$ is the nondimensional wall dilation rate defined to be positive for expansion and negative for contraction, and $\operatorname{Re}=a v_{w} / v$ is the filtration Reynolds number defined positive for injection and negative for suction through the walls. Equation (2.6) is strongly nonlinear and not easy to solve analytically, and most researchers have studied 
the classic Berman formula [8]; that is, when $\alpha=0$. In this paper, we seek to solve (2.6) subject to the boundary conditions (2.7) and (2.8) using a novel spectral modification of the homotopy analysis method and the successive linearisation method. By comparison with the numerical approximations and previously obtained results, we show that these new techniques are accurate and more efficient than the standard homotopy analysis method.

\section{Spectral Homotopy Analysis Method Solution}

In applying the spectral-homotopy analysis method, it is convenient to first transform the domain of the problem from $[0,1]$ to $[-1,1]$ and make the governing boundary conditions homogeneous by using the transformations

$$
y=\frac{\xi+1}{2}, \quad U(\xi)=F(y)-F_{0}(y), \quad F_{0}(y)=\frac{3}{2} y-\frac{1}{2} y^{3}
$$

Substituting (3.1) in the governing equation and boundary conditions (2.6)-(2.8) gives

$$
16 U^{1 V}+8 a_{1} U^{\prime \prime \prime}+4 a_{2} U^{\prime \prime}+2 a_{3} U^{\prime}-3 \operatorname{Re} U+8 \operatorname{Re}\left(U U^{\prime \prime \prime}-U^{\prime} U^{\prime \prime}\right)=\phi(y)
$$

subject to

$$
\begin{gathered}
U=0, \quad U^{\prime \prime}=0, \quad \xi=-1, \\
U=0, \quad U^{\prime}=0, \quad \xi=1,
\end{gathered}
$$

where the primes denote differentiation with respect to $\xi$ and

$$
\begin{gathered}
a_{1}=\alpha y+\operatorname{Re}\left(\frac{3}{2} y-\frac{1}{2} y^{3}\right), \quad a_{2}=3 \alpha-\frac{3}{2} \operatorname{Re}\left(1-y^{2}\right), \\
a_{3}=3 y \operatorname{Re}, \quad \phi(y)=12 \alpha y+3 \operatorname{Re} y^{3} .
\end{gathered}
$$

The initial approximation is taken to be the solution of the nonhomogeneous linear part of the governing equations (3.2) given by

$$
16 U_{0}^{1 V}+8 a_{1} U_{0}^{\prime \prime \prime}+4 a_{2} U_{0}^{\prime \prime}+2 a_{3} U_{0}^{\prime}-3 \operatorname{Re} U_{0}=\phi(y)
$$

subject to

$$
\begin{aligned}
& U_{0}=0, \quad U_{0}^{\prime \prime}=0, \quad \xi=-1, \\
& U_{0}=0, \quad U_{0}^{\prime}=0, \quad \xi=1 .
\end{aligned}
$$


We use the Chebyshev pseudospectral method to solve (3.5)-(3.6). The unknown function $U_{0}(\xi)$ is approximated as a truncated series of Chebyshev polynomials of the form

$$
U_{0}(\xi) \approx \mathrm{U}_{0}^{N}\left(\xi_{j}\right)=\sum_{k=0}^{N} \widehat{U}_{k} T_{k}\left(\xi_{j}\right), \quad j=0,1, \ldots, N
$$

where $T_{k}$ is the $k$ th Chebyshev polynomial, $\widehat{U}_{k}$, are coefficients and $\xi_{0}, \xi_{1}, \ldots, \xi_{N}$ are GaussLobatto collocation points (see [32]) defined by

$$
\xi_{j}=\cos \frac{\pi j}{N}, \quad j=0,1, \ldots, N
$$

Derivatives of the functions $U_{0}(\xi)$ at the collocation points are represented as

$$
\frac{d^{r} U_{0}}{d \xi^{r}}=\sum_{k=0}^{N} \Phi_{k j}^{r} U_{0}\left(\xi_{j}\right)
$$

where $r$ is the order of differentiation and $\Phi$ is the Chebyshev spectral differentiation matrix $([32,33])$. Substituting (3.7)-(3.9) in (3.5)-(3.6) yields

$$
\mathbf{A U}_{0}=\mathbf{\Phi},
$$

subject to the boundary conditions

$$
\begin{aligned}
U_{0}\left(\xi_{0}\right)=0, & U_{0}\left(\xi_{N}\right)=0, \\
\sum_{k=0}^{N} \Phi_{N k}^{2} U_{0}\left(\xi_{k}\right)=0, & \sum_{k=0}^{N} \Phi_{0 k} U_{0}\left(\xi_{k}\right)=0
\end{aligned}
$$

where

$$
\begin{aligned}
A & =16 \boldsymbol{\Phi}^{4}+8 \mathbf{a}_{1} \boldsymbol{\Phi}^{3}+4 \mathbf{a}_{2} \boldsymbol{\Phi}^{2}+2 \mathbf{a}_{3} \boldsymbol{\Phi}-3 \operatorname{Re} \mathbf{I}, \\
\mathbf{U}_{0} & =\left[U_{0}\left(\xi_{0}\right), U_{0}\left(\xi_{1}\right), \ldots, U_{0}\left(\xi_{N}\right)\right]^{T}, \\
\mathbf{\Phi} & =\left[\phi\left(y_{0}\right), \phi\left(y_{1}\right), \ldots, \phi\left(y_{N}\right)\right]^{T}, \\
\mathbf{a}_{s} & =\operatorname{diag}\left(\left[a_{s}\left(y_{0}\right), a_{s}\left(y_{1}\right), \ldots, a_{s}\left(y_{N-1}\right), a_{s}\left(y_{N}\right)\right]\right), \quad s=1,2,3 .
\end{aligned}
$$

In the above definitions, the superscript $T$ denotes transpose, diag is a diagonal matrix and I is an identity matrix of size $(N+1) \times(N+1)$.

To implement the boundary conditions (3.11), we delete the first and the last rows and columns of $A$ and delete the first and last rows of $\mathbf{U}_{0}$ and $\boldsymbol{\Phi}$. The boundary conditions (3.12) are imposed on the resulting first and last rows of the modified matrix $\mathbf{A}$ and 
setting the resulting first and last rows of the modified matrix $\mathbf{\Phi}$ to be zero. The values of $\left[U_{0}\left(\xi_{1}\right), U_{0}\left(\xi_{2}\right), \ldots, U_{0}\left(\xi_{N-1}\right)\right]$ are then determined from

$$
\mathbf{U}_{0}=\mathbf{A}^{-1} \boldsymbol{\Phi}
$$

To find the SHAM solutions of (3.2) we begin by defining the following linear operator:

$$
\left\llcorner[\tilde{U}(\xi ; q)]=16 \frac{\partial^{4} \tilde{U}}{\partial \xi^{4}}+8 a_{1} \frac{\partial^{3} \tilde{U}}{\partial \xi^{3}}+4 a_{2} \frac{\partial^{2} \tilde{U}}{\partial \xi^{2}}+2 a_{3} \frac{\partial \tilde{U}}{\partial \xi}-3 \operatorname{Re} \tilde{U},\right.
$$

where $q \in[0,1]$ is the embedding parameter, and $\tilde{U}(\xi ; q)$ is an unknown function.

The zeroth order deformation equation is given by

$$
\left.(1-q) \mathcal{L}\left[\tilde{U}(\xi ; q)-U_{0}(\xi)\right]=q \hbar\{\mathcal{N} \tilde{U}(\xi ; q)]-\mathbf{\Phi}\right\}
$$

where $\hbar$ is the nonzero convergence controlling auxiliary parameter and $\mathcal{N}$ is a nonlinear operator given by

$$
\mathcal{N}[\tilde{U}(\xi ; q)]=16 \frac{\partial^{4} \tilde{U}}{\partial \xi^{4}}+8 a_{1} \frac{\partial^{3} \tilde{U}}{\partial \xi^{3}}+4 a_{2} \frac{\partial^{2} \tilde{U}}{\partial \xi^{2}}+2 a_{3} \frac{\partial \tilde{U}}{\partial \xi}-3 \operatorname{Re} \tilde{U}+8 \operatorname{Re}\left(u \frac{\partial^{3} \tilde{U}}{\partial \xi^{3}}-\frac{\partial \tilde{U}}{\partial \xi} \frac{\partial^{2} \tilde{U}}{\partial \xi^{2}}\right) .
$$

Differentiating (3.17) $m$ times with respect to $q$ and then setting $q=0$ and finally dividing the resulting equations by $m$ ! yields the $m$ th order deformation equations

$$
\mathcal{L}\left[U_{m}(\xi)-\chi_{m} U_{m-1}(\xi)\right]=\hbar R_{m}
$$

subject to the boundary conditions

$$
U_{m}(-1)=U_{m}(1)=U_{m}^{\prime \prime}(-1)=U_{m}^{\prime}(1)=0,
$$

where

$$
\begin{aligned}
R_{m}(\xi)= & 16 U_{m-1}^{1 V}+8 a_{1} U_{m-1}^{\prime \prime \prime}+4 a_{2} U_{m-1}^{\prime \prime}+2 a_{3} U_{m-1}^{\prime}-3 \operatorname{Re} U_{m-1} \\
& +8 \operatorname{Re} \sum_{n=0}^{m-1}\left(U_{n} U_{m-1-n}^{\prime \prime \prime}-U_{n}^{\prime} U_{m-1-n}^{\prime \prime}\right)-\phi(y)\left(1-\chi_{m}\right), \\
X_{m}= & \begin{cases}0, & m \leq 1 \\
1, & m>1 .\end{cases}
\end{aligned}
$$

Applying the Chebyshev pseudospectral transformation on (3.19)-(3.21) gives

$$
\mathbf{A} \mathbf{U}_{m}=\left(x_{m}+\hbar\right) \mathbf{A} \mathbf{U}_{m-1}-\hbar\left(1-x_{m}\right) \mathbf{\Phi}+\hbar P_{m-1}
$$


subject to the boundary conditions

$$
\begin{aligned}
& U_{m}\left(\xi_{0}\right)=0, U_{m}\left(\xi_{N}\right)=0 \\
& \sum_{k=0}^{N} \Phi_{N k}^{2} U_{m}\left(\xi_{k}\right)=0, \quad \sum_{k=0}^{N} \Phi_{0 k} U_{m}\left(\xi_{k}\right)=0
\end{aligned}
$$

where $A$ and $\boldsymbol{\Phi}$, are as defined in (3.13) and

$$
\begin{aligned}
\mathbf{U}_{m} & =\left[U_{m}\left(\xi_{0}\right), U_{m}\left(\xi_{1}\right), \ldots, U_{m}\left(\xi_{N}\right)\right]^{T}, \\
P_{m-1} & =8 \operatorname{Re} \sum_{n=0}^{m-1}\left[U_{n}\left(\boldsymbol{\Phi}^{3} U_{m-1-n}\right)-\left(\Phi U_{n}\right)\left(\boldsymbol{\Phi}^{2} U_{m-1-n}\right)\right] .
\end{aligned}
$$

To implement the boundary conditions (3.24) we delete the first and last rows of $P_{m-1}$ and $\mathbf{\Phi}$ and delete the first and last rows and first and last columns of $\mathbf{A}$ in (3.23). The boundary conditions (3.25) are imposed on the first and last row of the modified A matrix on the left side of the equal sign in (3.23). The first and last rows of the modified A matrix on the right of the equal sign in (3.23) are the set to be zero. This results in the following recursive formula for $m \geq 1$ :

$$
\mathbf{U}_{m}=\left(\chi_{m}+\hbar\right) \mathbf{A}^{-1} \tilde{\mathbf{A}} \mathbf{U}_{m-1}+\hbar \mathbf{A}^{-1}\left[\mathbf{P}_{m-1}-\left(1-\chi_{m}\right) \boldsymbol{\Phi}\right]
$$

Thus, starting from the initial approximation, which is obtained from (3.15), higher-order approximations $U_{m}(\xi)$ for $m \geq 1$ can be obtained through the recursive formula (3.27).

\section{Successive Linearisation Method}

In this section, we solve (2.6) using the successive linearisation method. The main assumptions underpinning the use of the successive linearisation method are the following.

(i) The unknown function $F(y)$ maybe expanded as

$$
F(y)=F_{i}(y)+\sum_{m=0}^{i-1} F_{m}(y), \quad i=1,2,3, \ldots
$$

where $F_{i}$ are unknown functions and $F_{m}(m \geq 1)$ are approximations which are obtained by recursively solving the linear part of the equation that results from substituting (4.1) in the governing equation (2.6).

(ii) $F_{i}$ becomes progressively smaller as $i$ becomes large, that is,

$$
\lim _{i \rightarrow \infty} F_{i}=0
$$


Substituting (4.1) in the governing equation gives

$$
F_{i}^{(i v)}+a_{1, i-1} F_{i}^{\prime \prime \prime}+a_{2, i-1} F_{i}^{\prime \prime}+a_{3, i-1} F_{i}^{\prime}+a_{4, i-1} F_{i}+\operatorname{Re}\left(F_{i} F_{i}^{\prime \prime \prime}-F_{i}^{\prime} F_{i}^{\prime \prime}\right)=r_{i-1}
$$

where the coefficient parameters $a_{k, i-1},(k=1,2,3,4)$, and $r_{i-1}$ are defined as

$$
\begin{gathered}
a_{1, i-1}=\operatorname{Re} \sum_{m=0}^{i-1} F_{m}+\alpha y, \quad a_{2, i-1}=-\operatorname{Re} \sum_{m=0}^{i-1} F_{m}^{\prime}+3 \alpha, \\
a_{3, i-1}=-\operatorname{Re} \sum_{m=0}^{i-1} F_{m}^{\prime \prime}, \quad a_{4, i-1}=\operatorname{Re} \sum_{m=0}^{i-1} F_{m \prime}^{\prime \prime \prime} \\
r_{i-1}=-\left(\sum_{m=0}^{i-1} F_{m}^{(i v)}+\alpha y \sum_{m=0}^{i-1} F_{m}^{\prime \prime \prime}+3 \alpha \sum_{m=0}^{i-1} F_{m}^{\prime \prime}\right)-\operatorname{Re}\left(\sum_{m=0}^{i-1} F_{m} \sum_{m=0}^{i-1} F_{m}^{\prime \prime \prime}-\sum_{m=0}^{i-1} F_{m}^{\prime} \sum_{m=0}^{i-1} F_{m}^{\prime \prime}\right) .
\end{gathered}
$$

The SLM algorithm starts from the initial approximation

$$
F_{0}(y)=\frac{1}{2}(3+\beta) y-\frac{1}{2}(1+3 \beta) y^{3}+\beta y^{4}
$$

which is chosen to satisfy the boundary conditions (2.7)-(2.8). The parameter $\beta$ in (4.5) is an arbitrary constant which when varied results in multiple solutions. The subsequent solutions for $F_{m}, m \geq 1$ are obtained by successively solving the linearized form of (4.3) and which is given as

$$
F_{i}^{(i v)}+a_{1, i-1} F_{i}^{\prime \prime \prime}+a_{2, i-1} F_{i}^{\prime \prime}+a_{3, i-1} F_{i}^{\prime}+a_{4, i-1} F_{i}=r_{i-1}
$$

subject to the boundary conditions

$$
F_{i}(0)=0, \quad F_{i}^{\prime \prime}(0)=0, \quad F_{i}(1)=1, \quad F_{i}^{\prime}(1)=0 .
$$

Once each solution for $F_{i},(i \geq 1)$ has been found from iteratively solving (4.6) for each $i$, the approximate solution for $F(y)$ is obtained as

$$
F(y) \approx \sum_{m=0}^{M} F_{m}(y)
$$

where $M$ is the order of SLM approximation. Since the coefficient parameters and the right hand side of (4.6), for $i=1,2,3, \ldots$, are known from previous iterations, (4.6) can easily be solved using analytical means or any numerical methods such as finite differences, finite elements, Runge-Kutta-based shooting methods, or collocation methods. In this paper, (4.6) is integrated using the Chebyshev spectral collocation method $[32,33]$ as described in the previous section. 
Applying the Chebyshev spectral method to (4.6) leads to the matrix equation

$$
\mathbf{A}_{i-1} \mathbf{F}_{\mathbf{i}}=\mathbf{R}_{i-1}
$$

in which $\mathbf{A}$ is an $(N+1) \times(N+1)$ square matrix and $\mathbf{Y}$ and $\mathbf{R}$ are $(N+1) \times 1$ column vectors defined by

$$
\begin{gathered}
\mathbf{A}=\mathbf{D}^{4}+\mathbf{a}_{1, i-1} \mathbf{D}^{3}+\mathbf{a}_{2, i-1} \mathbf{D}^{2}+\mathbf{a}_{3, i-1} \mathbf{D}+\mathbf{a}_{4, i-1}, \\
\mathbf{R}_{i-1}=\mathbf{r}_{i-1},
\end{gathered}
$$

with

$$
\begin{gathered}
\mathbf{F}_{i}=\left[F_{i}\left(x_{0}\right), F_{i}\left(x_{1}\right), \ldots, F_{i}\left(x_{N-1}\right), F_{i}\left(x_{N}\right)\right]^{T}, \\
\mathbf{r}_{i-1}=\left[r_{i-1}\left(x_{0}\right), r_{i-1}\left(x_{1}\right), \ldots, r_{i-1}\left(x_{N-1}\right), r_{i-1}\left(x_{N}\right)\right]^{T} .
\end{gathered}
$$

In the above definitions, $N$ is the number of collocation points, $x=2 y-1, \mathbf{a}_{k, i-1},(k=1,2,3,4)$ are diagonal matrices of size $(N+1) \times(N+1)$, and $\mathbf{D}=2 \mathbf{2}$. After modifying the matrix system (4.9) to incorporate boundary conditions, the solution is obtained as

$$
\tilde{\mathbf{Y}}_{i}=\mathbf{A}_{i-1}^{-1} \mathbf{R}_{i-1}
$$

\section{Results and Discussion}

In this section, we compare the results obtained using the various methods: the SHAM, the SLM, and the numerical approximations with those obtained using the HAM in Dinarvand and Rashidi [30] and the homotopy-Páde method in Xu et al. [24]. The solution obtained using most numerical solutions depends on the initial approximation. Using different initial guesses can give rise to multiple solutions. Multiple solutions were obtained if the initial guess in (4.5) is used in the SHAM method in place of $F_{0}(y)$ in (3.1). In this paper, it was observed that using different values of $\beta$ results in multiple solutions. For the multiple solutions comparison was made against the HAM results of [24].

An optimal $\hbar$ value can easily be sought that can considerably improve the convergence rate of the results. However, for comparison purposes we used $\hbar=-1$. It is however worth noting, as pointed out in Dinarvand et al. [2], that when $\hbar=-1$, the solution series obtained by the HAM is the same solution series obtained by means of the homotopy perturbation method.

In Table 1 we compare the values of $F(y)$ when $\alpha=-1$ and $\operatorname{Re}=-2,0$ and 2 with the numerical and the HAM results reported in Dinarvand and Rashidi [30]. In [30], convergence up to six decimal places was achieved at the sixth order of the HAM approximation for $\mathrm{Re}=0$ and 2. In this study, the same level of convergence and accuracy was achieved at the first order approximation for the same values of Re. For $\operatorname{Re}=-2$ the convergence of the homotopy analysis method series solution was achieved at the eighth order of approximation while with the spectral homotopy analysis method series solution gives the same level of convergence at the second order. 
Table 1: Comparison of the numerical results against the SHAM approximate solutions for $F(y)$ when $\alpha=-1$ with $N=60$ and $\hbar=-1$.

\begin{tabular}{cccccccc}
\hline $\operatorname{Re}$ & $y$ & 0th order & 1st order & 2nd order & 3rd order & Numerical & Ref. [30] \\
\hline-2 & 0.2 & 0.273828 & 0.273831 & 0.273832 & 0.273832 & 0.273832 & 0.273832 \\
& 0.4 & 0.532827 & 0.532839 & 0.532839 & 0.532839 & 0.532839 & 0.532839 \\
& 0.6 & 0.759442 & 0.759467 & 0.759468 & 0.759468 & 0.759468 & 0.759468 \\
& 0.8 & 0.928967 & 0.928990 & 0.928990 & 0.928990 & 0.928990 & 0.928990 \\
\hline 0 & 0.2 & 0.279449 & 0.279449 & 0.279449 & 0.279449 & 0.279449 & 0.279449 \\
& 0.4 & 0.542243 & 0.542243 & 0.542243 & 0.542243 & 0.542243 & 0.542243 \\
& 0.6 & 0.768950 & 0.768950 & 0.768950 & 0.768950 & 0.768950 & 0.768950 \\
& 0.8 & 0.933889 & 0.933889 & 0.933889 & 0.933889 & 0.933889 & 0.933889 \\
\hline 2 & 0.2 & 0.283996 & 0.283983 & 0.283983 & 0.283983 & 0.283983 & 0.283983 \\
& 0.4 & 0.549759 & 0.549738 & 0.549738 & 0.549738 & 0.549738 & 0.549738 \\
& 0.6 & 0.776328 & 0.776306 & 0.776306 & 0.776306 & 0.776306 & 0.776306 \\
& 0.8 & 0.937518 & 0.937507 & 0.937507 & 0.937507 & 0.937507 & 0.937507 \\
\hline
\end{tabular}

Table 2: Comparison of the numerical results against the SHAM approximate solutions for $F^{\prime \prime}(1)$ when $\alpha=-1$ with $N=60$ and $\hbar=-1$ for different values of Re.

\begin{tabular}{lcccccc}
\hline $\operatorname{Re}$ & 0th order & 1st order & 2nd order & 3rd order & 4th order & Numerical \\
\hline 0 & -3.8213722 & -3.8213723 & -3.8213723 & -3.8213723 & -3.8213723 & -3.8213723 \\
5 & -3.1725373 & -3.1731774 & -3.1731800 & -3.1731800 & -3.1731800 & -3.1731800 \\
10 & -2.9069253 & -2.9069653 & -2.9069654 & -2.9069654 & -2.9069654 & -2.9069654 \\
15 & -2.7783086 & -2.7784366 & -2.7784369 & -2.7784369 & -2.7784369 & -2.7784369 \\
20 & -2.7056150 & -2.7060556 & -2.7060557 & -2.7060557 & -2.7060557 & -2.7060557 \\
25 & -2.6596223 & -2.6603920 & -2.6603907 & -2.6603907 & -2.6603907 & -2.6603907 \\
30 & -2.6281102 & -2.6291718 & -2.6291682 & -2.6291682 & -2.6291682 & -2.6291682 \\
40 & -2.5879160 & -2.5894333 & -2.5894247 & -2.5894247 & -2.5894247 & -2.5894247 \\
50 & -2.5634446 & -2.5652868 & -2.5652732 & -2.5652733 & -2.5652733 & -2.5652733 \\
100 & -2.5139141 & -2.5165255 & -2.5164964 & -2.5164967 & -2.5164967 & -2.5164967 \\
150 & -2.4972813 & -2.5001845 & -2.5001479 & -2.5001484 & -2.5001484 & -2.5001484 \\
\hline
\end{tabular}

In Table 2, we demonstrate the computational efficiency of the SHAM solution for large values of Re. As has been noted in the introduction, some semi-analytical methods fail to converge at large values of Re, for example, Dinarvand et al. [2] have shown that for $|\operatorname{Re}|>9.5$ the HPM fails to converge. However, in using the SHAM convergence up seven decimal places is achieved at the third order of approximation for values of Re as large as $\operatorname{Re}=150$. For $5 \leq \operatorname{Re}<100$ convergence up six decimal places is achieved at the second order. In Table $3, \operatorname{Re}=2$ is fixed and $F^{\prime \prime}(1)$ evaluated for $-2.5 \leq \alpha \leq 2.5$. Convergence up to seven digits is achieved at the first order for $\alpha=0$, at the second order for $-2 \leq \alpha \leq-0.5$, and at the third-order approximation for $\alpha=-2.5$. Clearly the SHAM gives faster convergence than the HAM under the same conditions.

Table 3 also gives a comparison of the SHAM approximate results with the numerical results generated using different values of $N$. It is evident however that for small values of $N$ (say less than $N=20$ ) the SHAM results are not very accurate. Accuracy however improves with an increase in $N$.

Table 4 gives a comparison of the numerical and the SHAM results of $F^{\prime \prime}(1)$ when $-2.5 \leq \alpha \leq 2.5$. Convergence is generally achieved at either the third- or the fourth-order 
Table 3: Comparison of the numerical results against the SHAM approximate solutions for $F^{\prime \prime}(1)$ when $\alpha=-1$ with $\operatorname{Re}=10$ and $\hbar=-1$ for different values of $N$.

\begin{tabular}{lcccccc}
\hline$N$ & 0th order & 1st order & 2nd order & 3rd order & 4th order & Numerical \\
\hline 10 & -2.7059921 & -2.7064302 & -2.7064302 & -2.7064302 & -2.7064302 & -2.7060557 \\
15 & -2.7056141 & -2.7060549 & -2.7060550 & -2.7060549 & -2.7060549 & -2.7060557 \\
20 & -2.7056150 & -2.7060556 & -2.7060557 & -2.7060557 & -2.7060557 & -2.7060557 \\
30 & -2.7056150 & -2.7060556 & -2.7060557 & -2.7060557 & -2.7060557 & -2.7060557 \\
40 & -2.7056150 & -2.7060556 & -2.7060557 & -2.7060557 & -2.7060557 & -2.7060557 \\
60 & -2.7056150 & -2.7060556 & -2.7060557 & -2.7060557 & -2.7060557 & -2.7060557 \\
\hline
\end{tabular}

Table 4: Comparison of the numerical results against the SHAM approximate solutions for $F^{\prime \prime}(1)$ when $\operatorname{Re}=2$ with $N=60$ and $\hbar=-1$ for different values of $\alpha$.

\begin{tabular}{lccccc}
\hline$\alpha$ & 1st order & 2nd order & 3rd order & 4th order & Numerical \\
\hline-2.5 & -4.5258487 & -4.5258506 & -4.5258505 & -4.5258505 & -4.5258505 \\
-2.0 & -4.1673848 & -4.1673892 & -4.1673892 & -4.1673892 & -4.1673892 \\
-1.5 & -3.8209704 & -3.8209740 & -3.8209740 & -3.8209740 & -3.8209740 \\
-1.0 & -3.4873966 & -3.4873982 & -3.4873982 & -3.4873982 & -3.4873982 \\
-0.5 & -3.1674332 & -3.1674334 & -3.1674334 & -3.1674334 & -3.1674334 \\
0.0 & -2.8618116 & -2.8618116 & -2.8618116 & -2.8618116 & -2.8618116 \\
0.5 & -2.5712067 & -2.5712055 & -2.5712055 & -2.5712055 & -2.5712056 \\
1.0 & -2.2962176 & -2.2962075 & -2.2962076 & -2.2962076 & -2.2962076 \\
1.5 & -2.0373492 & -2.0373088 & -2.0373092 & -2.0373092 & -2.0373092 \\
2.0 & -1.7949940 & -1.7948795 & -1.7948810 & -1.7948810 & -1.7948810 \\
2.5 & -1.5694172 & -1.5691503 & -1.5691557 & -1.5691556 & -1.5691556 \\
\hline
\end{tabular}

of the SHAM approximation. Figures 1 and 2 give a comparison of the numerical and the SHAM solutions for the characteristic mean-flow function $F(y)=-v / c$ and $F^{\prime}(y)=\mu c / x$ at different Reynolds numbers and $\alpha$. The mean-flow function $F(y)$, increases with increasing (positive) values of $\operatorname{Re}$ and $\alpha$ while $F^{\prime}(y)$ decreases, which makes sense since $F(y)$ is inversely proportional to the injection or suction coefficient $c=\alpha / \operatorname{Re}$ while $F^{\prime}(y)$ is directly proportional. Figure 1 further illustrates the efficiency of the solution method with excellent agreement for Re as large as 200.

Using the initial approximation $F_{0}(y)$ in (4.5) with different values of $\beta$ in place of (3.1) in the SHAM implementation leads to multiple solutions. When $\beta=0$ and -5 , the SHAM gives the multiple solutions observed in $\mathrm{Xu}$ et al. [24]. A comparison of the SHAM results against the HAM results reported in [24] is presented in Table 5. It is evident that the SHAM results converge much more rapidly than the HAM results of [24] for both branches of the solution.

Tables 6 and 7 give, first, the analytical approximations of $F^{\prime}(0) / \operatorname{Re}$ and $F^{\prime \prime \prime}(0) / \operatorname{Re}$ for the two solutions obtained using the successive linearisation method. Secondly, the tables give a direct comparison of the convergence rates of the SLM and the $[m, m]$ homotopy-Padé method used by $\mathrm{Xu}$ et al. [24]. The fourth-order SLM approximation gives the same level of accuracy as the twenty-fourth-order of the $[m, m]$ homotopy-Páde approximation, which suggests that the successive linearisation method is much more computationally efficient and accurate compared to the $[m, m]$ homotopy-Páde approximation (although it is not clear at this stage whether this could be attributed to the use of a more efficient initial guess). 


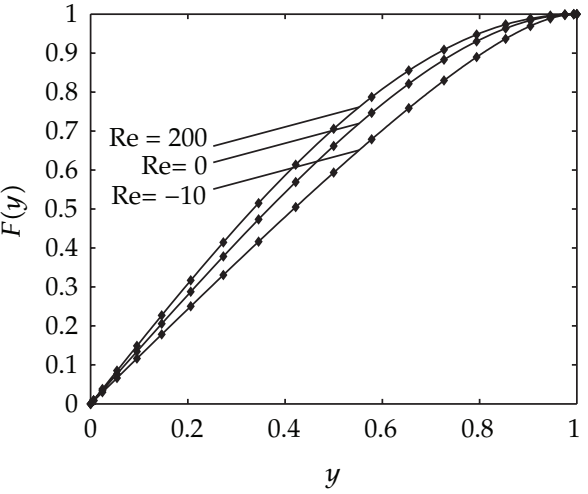

(a)



(b)

Figure 1: Comparison between numerical and SHAM approximate solution of $F(y)$ and $F^{\prime}(y)$ for different values of $\operatorname{Re}$ when $\alpha=-1$ when $\hbar=-1.14$ (for $\operatorname{Re}=-10$ ) and $\hbar=-1$ (for $\operatorname{Re}=0,200)$.



(a)



(b)

Figure 2: Comparison between numerical and SHAM approximate solution of $F(y)$ and $F^{\prime}(y)$ for different values of $\alpha$ when $\operatorname{Re}=2$ when $\hbar=-1$ (for $\alpha=-5,-2.5,0,2.5)$ and $\hbar=-0.94($ for $\alpha=5)$.

Table 5: Comparison between the multiple solutions of HAM results (see [24]) and the present SHAM results in the case of $\operatorname{Re}=-10$ and $\alpha=4$.

\begin{tabular}{lcccccc}
\hline & \multicolumn{3}{c}{ HAM solution [24] } & \multicolumn{3}{c}{ SHAM solution } \\
& Order of approx. & $F^{\prime}(0)$ & $F^{\prime \prime \prime}(0)$ & Order of approx. & $F^{\prime}(0)$ & $F^{\prime \prime \prime}(0)$ \\
\hline First solution & 10 & 0.624161 & 8.26756 & 5 & 0.625549 & 8.24662 \\
& 20 & 0.624967 & 8.25603 & 10 & 0.625016 & 8.25532 \\
& 30 & 0.625005 & 8.25548 & 15 & 0.625008 & 8.25544 \\
& 40 & 0.625007 & 8.25545 & 20 & 0.625007 & 8.25545 \\
Second solution & 50 & 0.625007 & 8.25545 & 25 & 0.625007 & 8.25545 \\
& 10 & -1.18995 & 35.8984 & 5 & -1.190529 & 35.85647 \\
& 20 & -1.19003 & 35.8474 & 10 & -1.190322 & 35.85414 \\
& 30 & -1.19041 & 35.8555 & 15 & -1.190323 & 35.85416 \\
& 40 & -1.19031 & 35.8539 & 20 & -1.190323 & 35.85416 \\
& 50 & -1.19033 & 35.8542 & 25 & -1.190323 & 35.85416 \\
\hline
\end{tabular}


Table 6: Comparison of $F^{\prime}(0) / \operatorname{Re}$ and $F^{\prime \prime \prime}(0) /$ Re obtained at different orders for the SLM, and the $[m, m]$ homotopy-Páde approximation when $\operatorname{Re}=-10$ and $\alpha=4$. For the SLM first solution, $\beta=-1$, and $N=25$, and for the SLM second solution, $\beta=-5$, and $N=25$.

\begin{tabular}{lcccccc}
\hline & \multicolumn{3}{c}{$[m, m]$ homotopy-Padé $[24]$} & \multicolumn{2}{c}{ SLM } \\
& order & $F^{\prime}(0) / \operatorname{Re}$ & $F^{\prime \prime \prime}(0) / \operatorname{Re}$ & order & $F^{\prime}(0)$ & $F^{\prime \prime \prime}(0)$ \\
\hline First solution & 4 & 0.624478732 & 8.265444222 & 2 & 0.624485895 & 8.26283765 \\
& 8 & 0.625005336 & 8.255477422 & 3 & 0.625007516 & 8.25544430 \\
& 16 & 0.625007395 & 8.255446127 & 4 & 0.625007396 & 8.25544612 \\
& 20 & 0.625007396 & 8.255446125 & 6 & 0.625007396 & 8.25544612 \\
Second solution & 24 & 0.625007396 & 8.255446124 & 8 & 0.625007396 & 8.25544612 \\
& 4 & -1.219891 & 36.01091 & 2 & -1.190934 & 35.86042 \\
& 16 & -1.178465 & 35.17878 & 3 & -1.190323 & 35.85416 \\
& 20 & -1.190319 & 35.85409 & 4 & -1.190323 & 35.85416 \\
& 24 & -1.190323 & 35.85415 & 6 & -1.190323 & 35.85416 \\
& & -1.190323 & 35.85416 & 8 & -1.190323 & 35.85416 \\
\hline
\end{tabular}

Table 7: Comparison of $F^{\prime}(0) /$ Re and $F^{\prime \prime \prime}(0) /$ Re obtained at different orders for the SLM, and the $[m, m]$ homotopy-Páde approximation when $\operatorname{Re}=-11$ and $\alpha=3 / 4$. For the SLM first solution, $\beta=-5$, and $N=25, \beta=1$, and $N=25$ for the SLM second solution, and for the SLM third solution $\beta=3$, and $N=25$.

\begin{tabular}{lcccccc}
\hline & \multicolumn{3}{c}{$[m, m]$ homotopy-Páde $[24]$} & \multicolumn{2}{c}{ SLM } \\
& order & $F^{\prime}(0) / \operatorname{Re}$ & $F^{\prime \prime \prime}(0) /$ Re & order & $F^{\prime}(0)$ & $F^{\prime \prime \prime}(0)$ \\
\hline First solution & 4 & -1.0231621 & 24.2925851 & 2 & -1.3250168 & 27.8640486 \\
& 8 & -1.0237700 & 24.2863797 & 3 & -1.0765777 & 24.9095006 \\
& 16 & -1.0237712 & 24.2863088 & 4 & -1.0259527 & 24.3119987 \\
& 20 & -1.0237712 & 24.2863088 & 6 & -1.0237712 & 24.2863088 \\
& 24 & -1.0237712 & 24.2863088 & 8 & -1.0237712 & 24.2863088 \\
\hline Second solution & 4 & 0.1668590 & 10.282860 & 2 & 0.2134950 & 9.682581 \\
& 8 & 0.1679980 & 10.239451 & 3 & 0.1718420 & 10.216566 \\
& 16 & 0.1693518 & 10.245026 & 4 & 0.1693573 & 10.245102 \\
& 20 & 0.1693532 & 10.245150 & 6 & 0.1693531 & 10.245151 \\
Third solution & 24 & 0.1693532 & 10.245151 & 8 & 0.1693531 & 10.245151 \\
\hline & 4 & $\ldots$ & $\ldots$ & 2 & 2.76262 & -15.5266 \\
& 8 & 2.81591 & -15.8950 & 3 & 2.76111 & -15.5123 \\
& 16 & 2.76154 & -15.5157 & 4 & 2.76111 & -15.5122 \\
& 20 & 2.76113 & -15.5123 & 6 & 2.76111 & -15.5122 \\
& 24 & 2.76111 & -15.5123 & 8 & 2.76111 & -15.5122 \\
\hline
\end{tabular}

\section{Conclusion}

In this paper, we have used the spectral homotopy analysis method (SHAM) and the successive linearisation method (SLM) to solve a fourth-order nonlinear boundary value problem that governs the two-dimensional Laminar flow between two moving porous walls. Multiple solutions recently reported in $\mathrm{Xu}$ et al. [24] have been obtained, depending on the initial approximation used. Comparison of the computational efficiency and accuracy of the results between the current methods and the previous homotopy analysis method results described in Dinarvand and Rashidi [30] and Xu et al. [24] has been made. Our simulations show that the convergence of the SHAM solution series to the numerical solution (up to six 
decimal place accuracy) is achieved at the second order (for $\operatorname{Re}=-2$ ) and first order for $R=0$, 2 . In contrast to the standard homotopy analysis method (c.f. Dinarvand and Rashidi [30]) convergence was achieved at the eighth order (for $\operatorname{Re}=-2$ ) and sixth order for $\operatorname{Re}=0,2$. The SHAM is apparently more efficient because it offers more flexibility in choosing linear operators compared to the standard HAM. It is however important to note that if the same initial guess and linear operators were to be used, the two methods would give the same solution.

We have further shown that notwithstanding the acceleration of the convergence ratio of the homotopy series by means of the homotopy-Pade technique, the successive linearisation techniques is more computationally efficient (although this again could be due, in part, to the use of a different initial guess) and gives accurate results.

\section{Acknowledgments}

The authors wish to acknowledge financial support from the University of KwaZulu-Natal and the National Research Foundation (NRF). The authers also thank the anonymous reviewers whose helpful comments have contributed to the improvement of our work.

\section{References}

[1] E. C. Dauenhauer and J. Majdalani, "Exact self-similarity solution of the Navier-Stokes equations for a porous channel with orthogonally moving walls," Physics of Fluids, vol. 15, no. 6, pp. 1485-1495, 2003.

[2] S. Dinarvand, A. Doosthoseini, E. Doosthoseini, and M. M. Rashidi, "Comparison of HAM and HPM methods for Berman's model of two-dimensional viscous flow in porous channel with wall suction or injection," Advances in Theoretical and Applied Mechanics, vol. 1, no. 7, pp. 337-347, 2008.

[3] J. Majdalani, "The oscillatory channel flow with arbitrary wall injection," Zeitschrift für Angewandte Mathematik und Physik, vol. 52, no. 1, pp. 33-61, 2001.

[4] J. Majdalani and T.-S. Roh, "The oscillatory channel flow with large wall injection," Proceedings of the Royal Society of London. Series A, vol. 456, no. 1999, pp. 1625-1657, 2000.

[5] J. Majdalani and W. K. van Moorhem, "Multiple-scales solution to the acoustic boundary layer in solid rocket motors," Journal of Propulsion and Power, vol. 13, no. 2, pp. 186-193, 1997.

[6] J. Majdalani and C. Zhou, "Moderate-to-large injection and suction driven channel flows with expanding or contracting walls," Zeitschrift für Angewandte Mathematik und Mechanik, vol. 83, no. 3, pp. 181-196, 2003.

[7] L. Oxarango, P. Schmitz, and M. Quintard, "Laminar flow in channels with wall suction or injection: a new model to study multi-channel filtration systems," Chemical Engineering Science, vol. 59, no. 5, pp. 1039-1051, 2004.

[8] A. S. Berman, "Laminar flow in channels with porous walls," Journal of Applied Physics, vol. 24, pp. 1232-1235, 1953.

[9] J. F. Brady, "Flow development in a porous channel and tube," Physics of Fluids, vol. 27, no. 5, pp. 1061-1076, 1984.

[10] S. M. Cox, "Two-dimensional flow of a viscous fluid in a channel with porous walls," Journal of Fluid Mechanics, vol. 227, pp. 1-33, 1991.

[11] S. P. Hastings, C. Lu, and A. D. MacGillivray, "A boundary value problem with multiple solutions from the theory of laminar flow," SIAM Journal on Mathematical Analysis, vol. 23, no. 1, pp. 201-208, 1992.

[12] T. A. Jankowski and J. Majdalani, “Symmetric solutions for the oscillatory channel flow with arbitrary suction," Journal of Sound and Vibration, vol. 294, no. 4, pp. 880-893, 2006.

[13] T. A. Jankowski and J. Majdalani, "Laminar flow in a porous channel with large wall suction and a weakly oscillatory pressure," Physics of Fluids, vol. 14, no. 3, pp. 1101-1110, 2002.

[14] C. Lu, "On the asymptotic solution of laminar channel flow with large suction," SIAM Journal on Mathematical Analysis, vol. 28, no. 5, pp. 1113-1134, 1997. 
[15] R. M. Terrill, "Laminar flow in a uniformly porous channel," The Aeronautical Quarterly, vol. 15, pp. 299-310, 1964.

[16] S. Uchida and H. Aoki, “Unsteady flows in a semi-infinite contracting or expanding pipe," Journal of Fluid Mechanics, vol. 82, no. 2, pp. 371-387, 1977.

[17] C. Zhou and J. Majdalani, "Improved mean-flow solution for slab rocket motors with regressing walls," Journal of Propulsion and Power, vol. 18, no. 3, pp. 703-711, 2002.

[18] G. Adomian, "Nonlinear stochastic differential equations," Journal of Mathematical Analysis and Applications, vol. 55, no. 2, pp. 441-452, 1976.

[19] G. Adomian, "A review of the decomposition method and some recent results for nonlinear equations," Computers \& Mathematics with Applications, vol. 21, no. 5, pp. 101-127, 1991.

[20] J.-H. He, "A coupling method of a homotopy technique and a perturbation technique for non-linear problems," International Journal of Non-Linear Mechanics, vol. 35, no. 1, pp. 37-43, 2000.

[21] J.-H. He, "Homotopy perturbation method for solving boundary value problems," Physics Letters A, vol. 350 , no. 1-2, pp. 87-88, 2006.

[22] S. J. Liao, The proposed homotopy analysis technique for the solution of nonlinear problems, Ph.D. thesis, Shanghai Jiao Tong University, 1992.

[23] S. Liao, Beyond Perturbation: Introduction to Homotopy Analysis Method, vol. 2 of CRC Series: Modern Mechanics and Mathematics, Chapman \& Hall/CRC, Boca Raton, Fla, USA, 2004.

[24] H. Xu, Z. L. Lin, S. J. Liao, J. Z. Wu, and J. Majdalani, "Homotopy based solutions of the Navier-Stokes equations for a porous channel with orthogonally moving walls," Physics of Fluids, vol. 22, Article ID 053601, 18 pages, 2010.

[25] M. B. Zaturska, P. G. Drazin, and W. H. H. Banks, “On the flow of a viscous fluid driven along a channel by suction at porous walls," Fluid Dynamics Research, vol. 4, no. 3, pp. 151-178, 1988.

[26] S. S. Motsa, P. Sibanda, and S. Shateyi, "A new spectral-homotopy analysis method for solving a nonlinear second order BVP," Communications in Nonlinear Science and Numerical Simulation, vol. 15, no. 9, pp. 2293-2302, 2010.

[27] S. S. Motsa, P. Sibanda, F. G. Awad, and S. Shateyi, "A new spectral-homotopy analysis method for the MHD Jeffery-Hamel problem," Computers and Fluids, vol. 39, no. 7, pp. 1219-1225, 2010.

[28] Z. Makukula, S. S. Motsa, and P. Sibanda, "On a new solution for the viscoelastic squeezing flow between two parallel plates," Journal of Advanced Research in Applied Mathematics, vol. 2, no. 4, pp. 31-38, 2010

[29] S. S. Motsa and P. Sibanda, "A new algorithm for solving singular IVPs of Lane-Emden type," in Proceedings of the 4th International Conference on Applied Mathematics, Simulation, Modelling, WSEAS International Conferences, pp. 176-180, Corfu Island, Greece, July 2010.

[30] S. Dinarvand and M. M. Rashidi, "A reliable treatment of a homotopy analysis method for twodimensional viscous flow in a rectangular domain bounded by two moving porous walls," Nonlinear Analysis: Real World Applications, vol. 11, no. 3, pp. 1502-1512, 2010.

[31] J. Majdalani, C. Zhou, and C. A. Dawson, "Two-dimensional viscous flow between slowly expanding or contracting walls with weak permeability," Journal of Biomechanics, vol. 35, no. 10, pp. 1399-1403, 2002.

[32] C. Canuto, M. Y. Hussaini, A. Quarteroni, and T. A. Zang, Spectral Methods in Fluid Dynamics, Springer Series in Computational Physics, Springer, New York, NY, USA, 1988.

[33] L. N. Trefethen, Spectral Methods in MATLAB, vol. 10 of Software, Environments, and Tools, SIAM, Philadelphia, Pa, USA, 2000. 




Advances in

Operations Research

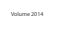

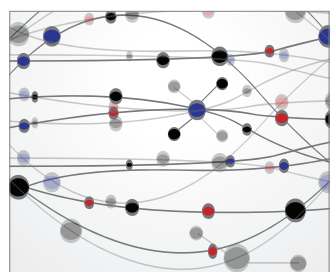

\section{The Scientific} World Journal


International Journal of

Mathematics and

Mathematical

Sciences
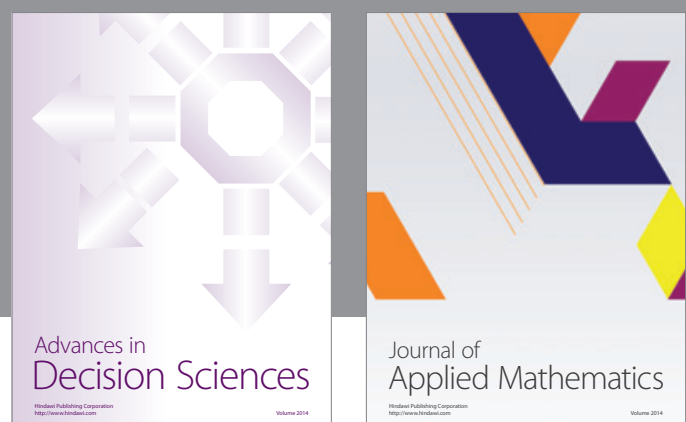

Journal of

Applied Mathematics


Submit your manuscripts at http://www.hindawi.com


Mathematical Problems in Engineering


Journal of

Function Spaces




ournal of

Probability and Statistics

Promensencen
